\title{
Representing Statics as Forces in Equilibrium
}

\author{
Jennifer J. Freyd and Teresa M. Pantzer \\ University of Oregon
}

Jeannette L. Cheng

University of Delaware

\begin{abstract}
SUMMARY
Resting objects can be described according to the physical forces operating on them, forces that are balanced in static scenes. We hypothesized that in a related way, the perception of static scenes and objects might involve a representation of underlying dynamics. In our first experiments, subjects were shown a picture of a plant resting upon a table or hanging from a hook, followed by a picture of the plant in the same position without the supporting table or hook. Subjects attempted to remember the position of the plant and were then shown a third display, in which the plant was in the same position or was slightly above or slightly below the original position. We found that subjects made more errors for test displays showing the plant slightly below, as compared with displays showing the plant slightly above, the original position. That is, memory for the position of the previously supported object was distorted in the direction consistent with what would happen if the plant was to lose its source of support in real life. This effect depends on the initial display of support; in Experiment 2 we found no memory asymmetry when the plant was initially displayed without support. We replicated the results of Experiments 1 and 2 with a new stimulus set and modified procedure in Experiment 3. In our fourth study we experimented with a slightly different stable situation: a spring with a box on top of it. We found that subjects misremembered the spring as either more compressed or less compressed as predicted by the implied dynamics of the display sequence. We discuss issues raised by our findings, including the possibility that the conscious experience of concreteness in static scenes stems from the representation of underlying forces.
\end{abstract}

Much of what people encounter in everyday life is static from their point of reference: Cups rest on desks, chairs sit on floors, and books stand on shelves. Perhaps it is the very pervasiveness of static objects and still scenes that has been responsible for psychology's historical focus on the perception of static qualities of the world: shape and form perception, pattern recognition, picture perception, and object recogni-

A series of experiments preliminary to those described in this article was reported by Jennifer J. Freyd and Jeanette L. Cheng at the 27th Annual Meeting of the Psychonomic Society, 1986. Most of the data reported here were collected when Freyd and Teresa $M$. Pantzer were at Cornell University. This research was supported by National Institute of Mental Health Grant BBP-MH39784 and National Science Foundation Presidential Young Investigator Award BNS-8451356 to Freyd.

We thank Robert Mauro for providing statistical advice and Asher Cohen, Paul Compton, Mark Faust, Morton Gernsbacher, Peggy Jennings, J. Q. Johnson, Steve Keele, Roger Shepard, Jim Tanaka, and Marjory Taylor for their comments on earlier drafts of this article. We also appreciate the suggestions for revision made by the editor, Sam Glucksberg, and the reviewers of this article (Julian Hochberg and an anonymous referee).

Correspondence concerning this article should be addressed to Jennifer J. Freyd, Department of Psychology, University of Oregon, Eugene, Oregon 97403. tion. In apparent contrast to this focus, there has been an increasingly popular emphasis on the perception of events, or patterns of change in the world. There is a sense, however, in which the study of event perception (e.g., J. J. Gibson, 1979) has shared some assumptions with the more traditional focus on the perception of static stimuli. In both approaches events and dynamic stimuli have been defined in terms of changes taking place in real time, whereas scenes that are not changing in real time (or are being viewed by an observer who is not moving in real time) have been considered simply static, that is, specifically not dynamic.

This view of static objects and scenes suggests that the perception of a static scene is devoid of information about dynamic qualities of the world (which led J. J. Gibson, 1970, for instance, to consider the perception of static scenes to be a mere laboratory curiosity). But, if we take dynamic to mean relating to physical force acting on objects with mass, then this view is incorrect. As physicists have maintained since Galileo, objects in the world are dynamic, even when they are not moving. Resting objects in the environment are constantly subject to forces, although those forces may be in equilibrium.

We conducted a series of experiments designed to demonstrate that the perception of static objects and scenes is in keeping with this physical description of objects. We came to this hypothesis, not through a consideration of Newtonian mechanics, but instead through empirical results suggesting 
that perceivers represent implied motion when viewing static snapshots of objects captured in motion (Freyd, 1983) and the recent theoretical argument that some, perhaps all, mental representations are dynamic (Freyd, 1987).

The empirical results we first considered were Freyd's (1983) finding that subjects represent the implied motion captured in photographs of moving scenes ("frozen-action" photographs). Freyd (1983) created pairs of frozen-action photographs by printing selected frames from short films of unidirectional action sequences (such as human and animal motion and natural processes moving toward a greater state of entropy). She selected a number of picture pairs corresponding to frames close together in the film. She then used these pairs in a simple task in which subjects were shown one member of the pair for $250 \mathrm{~ms}$, were given a $250-\mathrm{ms}$ retention interval, and were then shown the other member of the pair. The subjects' task was to indicate whether the second picture was exactly the same as or different from the first picture. For those cases in which the correct answer was different, one half of the pairs were shown in real-world, or forward, order, and the other half were shown in backward order (so that the picture printed from a frame early in the film was shown after a picture printed from later in the film). Freyd (1983) found that subjects had significantly longer reaction times for correctly indicating that the second picture was different from the first for the forward pairs, as compared with the backward pairs. Freyd (1983) concluded that subjects found the forward pairs more confusable than the backward pairs because the perception of the first picture in the pair involved a mental unfreezing of the frozen action in the forward direction.

Freyd's (1983) result suggested that the perception of static stimuli can involve the representation of dynamic information. However, the stimuli Freyd (1983) used in her experiment strongly implied motion, even though there was no motion in real time. We wondered if subjects might represent dynamic information even when the objects being portrayed were distinctly stable. One sort of dynamic information in such a case could be the forces acting on the pictured objects that are in balance.

It is difficult to design a paradigm that determines whether subjects represent static objects as acted on by forces in balance. We predict that subjects represent the stability of forces inherent in a static scene. Thus, the mental representation of the dynamics of the resting objects should be especially resistant to transformation. Results indicating that the representation does not lead to memory errors are potentially simply negative results that cannot support a rejection of the null hypothesis. This difficulty is in contrast to the logic Freyd (1983) used to investigate the representation of frozen-action photographs, in which such representations are hypothesized to be changing in a particular direction. Our solution, in the present studies, was to show subjects a static scene followed by the same scene in which a critical source of support was no longer present. We predicted that because in the second scene physical forces represented in the first scene were suddenly no longer in equilibrium, the removal of support would induce a mental representation that was changing in a particular direction. In other words, we predicted that because subjects represent static objects in terms of their forces, those forces would unfreeze the object when they were no longer in equilibrium and would lead to a tendency for subjects to misremember the position of an object in the direction of the net forces.

\section{Experiment 1}

The most common sort of static scene that people witness in everyday life involves one object resting on another. In such cases the force of gravity is counterbalanced by the normal force of the supporting surface. We used a display with this common combination of forces: a potted plant resting on a table. This permitted us to show first the plant on the table, then the plant unsupported, followed by a test picture of the plant in the same location or one slightly different from the original location. The first row of pictures in Figure 1 depicts the three displays used in a trial beginning with the plant resting on the table (accompanied by a picture of a window, intended to provide a reference point for subjects) and ending with a test display that shows the plant in the original position.

We planned to compare two distractor positions for the plant, one slightly higher than the original position and one slightly lower than the original position. Our prediction was that the lower distractor position would be harder to identify correctly as different from the higher distractor position, because the force of gravity would cause the plant to move downward once the table was removed. That is, we hypothesized that subjects would represent the balanced forces in the first display and would therefore represent the force of gravity as suddenly unopposed and therefore dynamically manifested in the second display.

We included one other type of trial in the first experiment: For every trial beginning with the plant resting on the table, there were an equal number of trials beginning with the plant hanging from a hook. The position of the plant with respect to the screen and the window depicted on the screen was exactly the same as that for displays showing the plant on the table and displays showing the plant hanging from the hook. Also, the second display (the plant alone) and the test displays for hook trials were identical to those used for the table trials. The second row of Figure 1 depicts a trial beginning with the plant on the hook and ending with the plant in the original position. The hook trials were included to control for a possible tendency to misremember the plant closer to the location of a previously shown object; in the hook trials we predicted that subjects would misremember the plant as farther away from the hook, not closer to it. We thus hoped to find that there was not a significant interaction between the source of support (hook or table) and the direction of displacement (up or down).

We chose our timing intervals (250-ms stimulus durations for the first and second displays and 250-ms interstimulus intervals between the first and second and between the second and third displays) on the basis of the times used in the experiments on the representation of implied motion in frozen-action pictures (Freyd, 1983). Departing from Freyd (1983), however, we measured error rate instead of reaction 


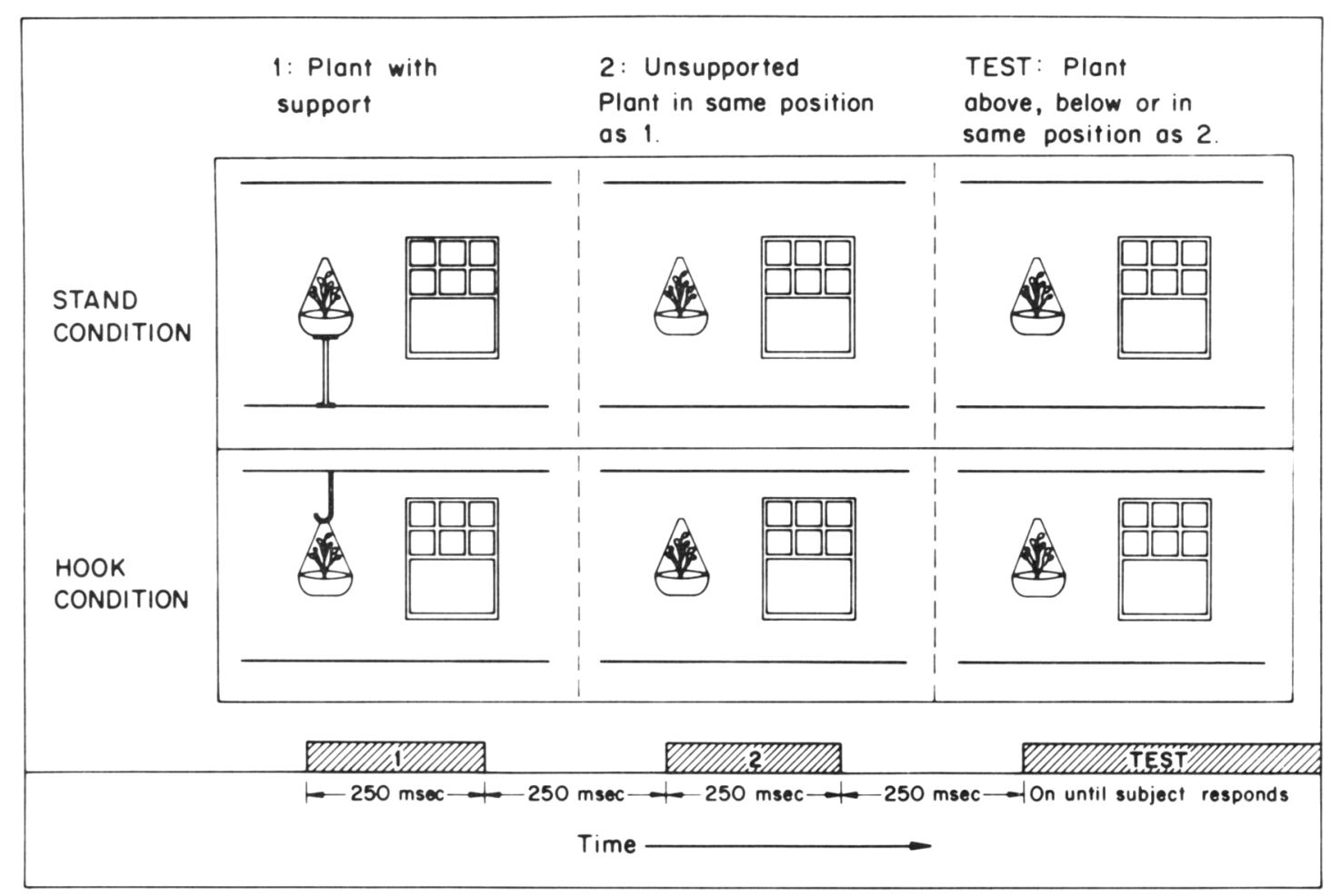

Figure 1. Schematic depiction of the stimuli used in Experiment 1. The first row shows the three displays used in a trial beginning with the table as a support object and ending with the test display showing the plant in the original position. The second row shows a comparable trial for the hook condition. Timing of displays and intervals is indicated at the bottom of the figure.

time (RT). This choice of measure followed experiments by Freyd and Finke and their co-workers (e.g., Freyd \& Finke, 1984, 1985; Freyd \& Johnson, 1987), in which memory distortions were induced by showing subjects a sequence of static positions of an object sampled from a path of rotation. Freyd and Finke (1984) found that both error rate and RT indicated that subjects misremembered the position of an object as farther away from its last location, along the direction of implied motion. Beginning with Freyd and Finke (1985), error rate became the preferred measure; in many conditions subjects made an insufficient number of correct responses to calculate a reliable RT. Pilot data collected on the present experiment suggested that we would have a similar difficulty in measuring RT with error rates as high as $50 \%$ in some conditions.

\section{Method}

Subjects. Twelve subjects, recruited from the Cornell University community, were paid for their participation in this experiment. Six of these subjects had previously been in related experiments in the same laboratory; six were new subjects. As expected, this betweensubjects difference yielded no significant effects.

Apparatus. Stimuli were presented on a Hewlett-Packard (HP) $1340 \mathrm{~A}$ vector-plotting display screen, $9.6 \mathrm{~cm}$ by $11.9 \mathrm{~cm}$. The display screen is connected by an HP 1351A graphics generator to an HP 9133A microcomputer. Subjects used a foot pedal and two separate key presses (one for each hand) to initiate trials and to indicate responses. Subjects sat at a comfortable viewing distance from the graphics screen, in a well-lit laboratory room.

Stimuli and format of trials. Figure 1 depicts the stimuli used in this experiment. At the start of each trial, subjects were presented with either a line drawing of a plant in a hanging planter standing on a table next to a window, as displayed in the first cell of Figure 1, or a picture of a plant hanging from a hook, as displayed in the first column of the second row of Figure 1. The length of the plant, including hanging planter, was $2.35 \mathrm{~cm}$. The position of the plant in each of the two source-of-support conditions (table or hook) was identical with respect to the computer screen and the line drawing of the window. The plant with its source of support remained on the screen for $250 \mathrm{~ms}$. The screen was then blank for $250 \mathrm{~ms}$, followed by the presentation of the plant without its source of support, shown for $250 \mathrm{~ms}$. This second picture showed the plant in exactly the same position it had been in previously. This was followed by a $250-\mathrm{ms}$ retention interval, in which subjects were asked to remember the exact position of the plant. The final display in each trial was the plant in the same position as in the first two displays or slightly raised or lowered by $0.14 \mathrm{~cm}$. The window was pictured in all displays to provide a reference point for subjects.

Procedure. Subjects were given thorough written and oral instruction. They were told about the makeup of individual trials and were asked to indicate if the plant in the third display was in exactly the same position as it had been in the second display. They were instructed to hit one key for same, and the other key for different. (Subjects were instructed to initiate each trial by pressing on a foot pedal.) Subjects were told that they should not expect an equal number of same and different trials. Both speed and accuracy were stressed in the instructions. In addition, subjects were instructed to 
attend to the whole picture and to attempt to avoid concentrating on the plant alone.

Each subject completed a critical block of 60 trials. These 60 trials were presented in a random order for each subject and were formed from 2 sources of support (table or hook) by 3 plant positions (up, same, or down) by 10 replications of each trial type.

While subjects were in the laboratory, they were asked to complete two additional blocks of 60 trials for a related experiment with a similar paradigm. The order of the three blocks of trials was counterbalanced across subjects, so that 2 subjects received each of the six possible orderings of three blocks. We did not expect the position of the critical block in the experimental session (first, second, or third) to result in a significant main effect or to interact with any other variable.

\section{Results and Discussion}

Error rates for each condition and each subject were calculated. Averaged across subjects, the error rate for true-same trials (in which the position of the final plant was in the same position as it had been in the first and second displays) was $8 \%$ for trials beginning with the plant on the table and $13 \%$ for trials beginning with the plant on the hook. The error rates for same trials were not significantly different for the two conditions.

An initial analysis of variance (ANOVA) was performed with four variables: Experience of subject (whether the subject had been in previous experiments in our laboratory) and position of critical block in the experimental session (first, second, or third) were between-subjects variables, and source of support (table or hook) and position of distractor (up or down) were within-subjects variables. As expected, neither between-subjects variable was significant, nor did they significantly interact with any other variables $(p>.34$ for both main effects and all interactions involving these between-subjects variables in the initial ANOVA). These variables were therefore dropped from all subsequent analyses.

Table 1 displays average error rates for the conditions of interest. An ANOVA was performed with error rates for the two between-subjects variables: source of support (table or hook) and position of distractor (up or down). There was neither a main effect for source of support nor an interaction between it and position of distractor (in both cases $F<1$, $p>$.46). There was, however, a main effect for position of distractor in the predicted direction: $F(1,11)=5.489, p=$ $.039, M S_{\mathrm{e}}=8.385$.

The predicted difference between up and down distractors was found in Experiment 1; subjects made significantly more errors when the plant was shown in a position lowered from

Table 1

Experiment 1: Error Rates and Averages (\%)

\begin{tabular}{cccc}
\hline & \multicolumn{2}{c}{ Test position } & \\
\cline { 2 - 4 } Source of support & Up & Down & Average \\
\hline Table & 39 & 58 & 48 \\
Hook & 35 & 56 & 45 \\
Average & 37 & 57 & \\
\hline
\end{tabular}

true-same than when it was shown in a position raised from true-same. This result was not contingent on the source of support (hook or table), suggesting that it does not stem from a tendency to misremember objects in positions previously occupied by other objects. We believe the results of Experiment 1 support the hypothesis that subjects represent the forces operating on static objects.

\section{Experiment 2}

In Experiment 1 we found that subjects misremembered the position of a previously supported plant in the direction predicted from the hypothesis where the force of gravity is represented. We were concerned, however, that we did not demonstrate that the initial stable display shown in each trial was necessary to the effect, despite our assumption that the memory effects arose from a representation of the forces in equilibrium that were suddenly unbalanced. We were also concerned that our finding might stem from a general tendency to misremember an object as further down on the screen, independent of a representation of forces. In Experiment 2 we answered these concerns by using a sequence of displays (see Figure 2) that did not indicate a sudden disruption of forces in equilibrium but were otherwise very similar to those used in Experiment 1. Such a display, we reasoned, should lead to no consistent memory errors, if our initial hypothesis was correct.

\section{Method}

Twelve additional subjects recruited from the Cornell University community were paid for their participation in this experiment. Again 6 of the 12 subjects had previously been in related experiments in the laboratory (but not Experiment 1), and 6 were new subjects, and again we did not expect this factor to lead to any significant effects. The methods used in Experiment 2 were very similar to those used in Experiment 1. The display sequence, depicted in Figure 2, was the same as that used in the first experiment except that the plant was never shown with support. Thus the subjects saw the plant next to the window, followed by the plant again next to the window, followed by the unmoved plant in either the same location or one slightly different. The second and third displays used in Experiment 2 were the same as those used in Experiment 1 . Subjects were tested in a critical block of 60 trials, formed of 20 replications each of trials ending with test items in the same position, slightly lower, or slightly higher. Again this critical block of trials was run with two additional blocks of trials that were similar in format but different in content. The order of blocks was counterbalanced across subjects as in Experiment 1 , and again we did not expect the position of the critical block within the experimental session to lead to any significant effects.

\section{Results and Discussion}

Error rates were calculated as in Experiment 1. Across subjects the error rate for true-same trials was $26 \%$; for up trials, 33\%; and for down trials, 34\%. As in the first experiment, initial analyses confirmed our expectation that the two between-subjects variables (experience of subject and position of block in the experimental session) did not lead to significant effects; they were dropped as variables from subsequent anal- 


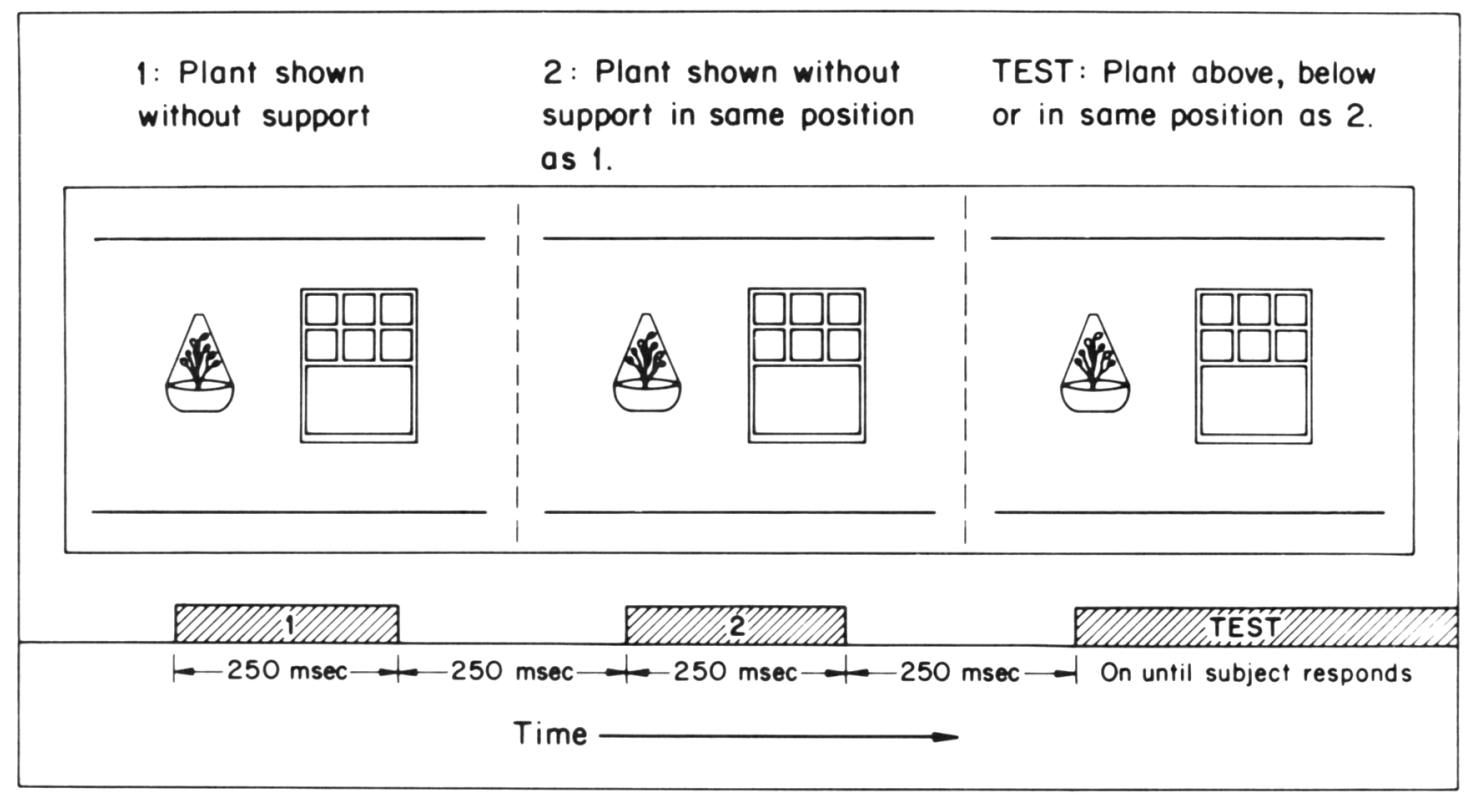

Figure 2. Schematic depiction of the stimuli used in Experiment 2. The three displays shown are from a trial ending with the test display showing the plant in the original position. Timing of displays and intervals is indicated at the bottom of the figure.

yses. A one-way ANOVA revealed that the within-subjects variable of direction of distractor positions (up or down) was not significant: $F(1,11)=.065, p=.803, M S_{\mathrm{e}}=5.739$.

It is evident from Figure 3, in which the results from Experiments 1 and 2 are shown together, that Experiment 2 was an effective control for the possibility that subjects simply misremember the plant as further down, independent of the forces implied by the sequence of displays. This conclusion is supported by a two-sample $t$ test comparing difference scores (up minus down error rates) for each subject in Experiment 1 with difference scores in Experiment 2, indicating a significant difference between the two experiments: $t(22)=1.893$, $p=.036$ (one-tailed).

The information presented in the initial display of Experiment 1 trials is sufficiently clear to induce mental representations that include forces and thus changes in the position of the plant. In contrast, the initial displays of Experiment 2 must be either sufficiently ambiguous regarding forces operating on the plant so that subjects do not represent the forces in any consistent manner, or the initial displays may indicate that the plant has a hidden source of support, so that subjects maintain a representation of the plant in a stable position throughout the trial.

\section{Experiment 3}

Experiments 1 and 2 taken together support the hypothesis that subjects represent the underlying dynamics of coherent static scenes, so that when the forces are suddenly unbalanced the mental representation includes an unfreezing of the potential motion implicit in the scene. In our third experiment we replicated and extended the first two experiments, with three changes: (a) We used a new display, (b) we varied the coherence of the static scene (whether support was shown initially) as a within-subjects variable, and (c) we used seven test positions, varied parametrically around the true-same position, allowing a finer determination of memory for position. This last change followed the method introduced by Freyd and Finke (1985) for studying memory errors in rep-

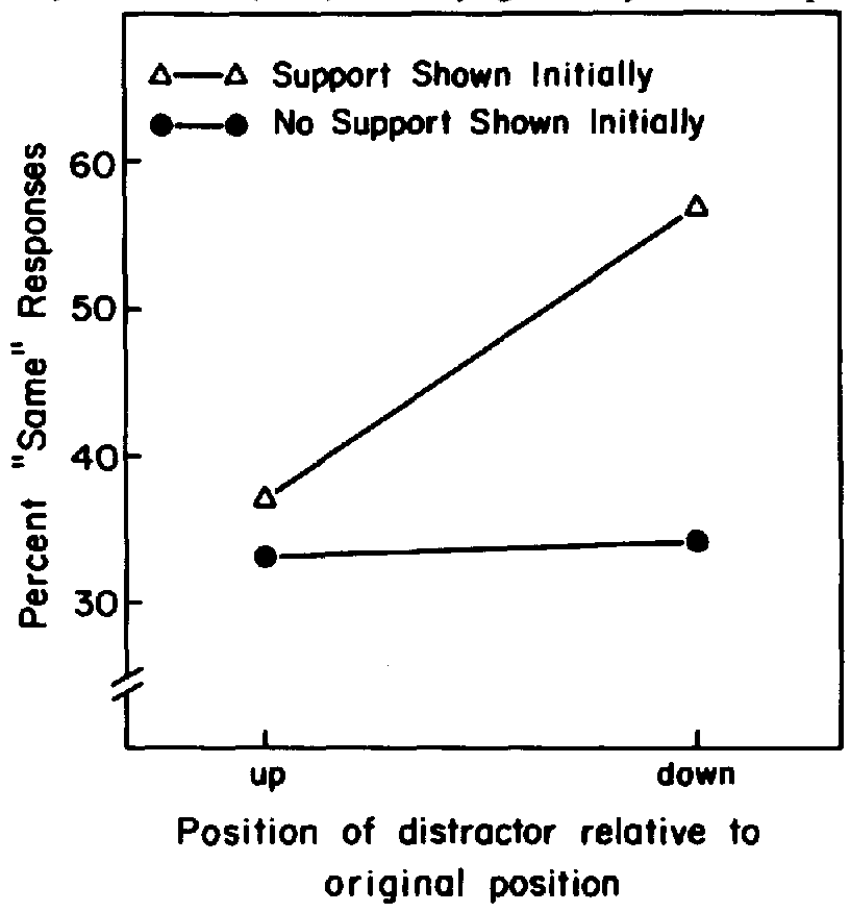

Figure 3. Error rates for trials ending with test displays showing the plant up versus down from Experiments 1 (support) and 2 (no support) are plotted together. 
resentational momentum experiments. A range of distractor positions allows one to estimate the amount of memory shift for a given condition and subject. We predicted that shifts would be significantly greater for the support-present condition than for the no-support condition.

We used a drawing of a lock hanging from a hook for the first display in our support-present condition (see Figure 4) and a lock presented alone for the first display in our nosupport condition. These two conditions were predicted to replicate the results of Experiment 1 and Experiment 2, respectively. We did not show subjects a drawing of a lock resting on a supporting object but used only the hanging condition (with a hook very large in proportion to the lock) as it was the more conservative test of our hypothesis. (It controlled for the possibility that people misremember objects as closer to previously occupied spaces.)

\section{Method}

Subjects. Seventeen subjects, recruited from the Cornell University community, were paid for their participation in this experiment. The results from 16 of these subjects were used in our analyses; the data from 1 subject were discarded because he reported switching the same and different buttons halfway through the experiment. None of the subjects had participated in Experiment 1 or 2. Some subjects had been in other experiments in the laboratory; we did not consider this a factor needing counterbalancing given the lack of effect for experience of subjects in Experiments 1 and 2.

Stimuli and procedure. Subjects received thorough written and oral instructions similar to the instructions used in the first two experiments. Individual trials followed the exact timing and ordering

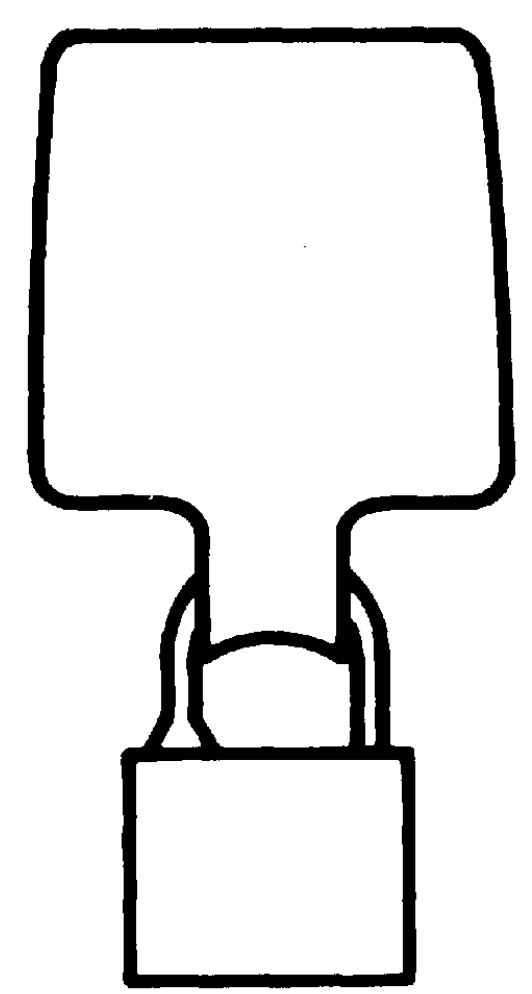

Figure 4. Schematic depiction of the first display used in supportpresent trials in Experiment 3. used in the first two experiments. In this experiment subjects received two blocks of 140 trials, each preceded by 21 practice trials. One block was formed of trials beginning with the lock supported by the hook; the other (control) block was formed of trials beginning with the lock shown without support. Otherwise, trials in the two blocks were the same. The 140 trials were formed of 20 replications of each of seven test positions. (One position was true-same; three were higher, and three were lower, in steps of $0.094 \mathrm{~cm}$ from true-same; the height of the lock was $2.73 \mathrm{~cm}$.) The 21 practice trials were formed of 3 replications of each of the seven test positions. Order of block was counterbalanced across subjects.

\section{Results and Discussion}

The percentage of same responses was calculated for each of the seven test positions for each of the two support conditions. Figure 5 shows mean percentage of same responses: The solid line connects data points for the support condition; the dashed line is for the no-support (or control) condition.

For each subject we calculated two shift values, one for the support-present condition and one for the no-support condition. We followed the technique introduced by Freyd and Finke (1985) for calculating shifts: The number of same responses for each of the seven distractor positions was entered into a quadratic regression using distractor position, ranging from -3 (the most upward distractor position) to +3 (the lowest distractor position), and distractor position squared as predictors. We solved the regression equation for the estimated peak in the curve and took the result as the estimated shift. A shift of -0.50 would mean that the curve peaks at a position midway between true-same and the first distractor position in the upward direction; a shift of +0.50 would mean the curve peaks at a position midway between true-same and the first downward distractor position. We predicted that shifts for the support-present condition should be positive and shifts for the no-support condition should be very close to 0.0 , that is, no consistent shift at all.

We found that for the support-present condition, the average shift (i.e., an average of individual subject shifts) was +0.283 . A $t$ test revealed that this value was significantly different from $0.0: t(15)=2.204, p=.044$ (two-tailed). The average shift for the no-support condition was +0.075 ; it was not significantly different from the null hypothesis of no shift, $t(15)=0.957, p=.354$. We also tested for a significant difference between the support-present and the no-support conditions in a two-way ANOVA in which block order was used as a between-subjects variable. The ANOVA revealed a significant difference between the support-present and the nosupport conditions: $F(1,14)=5.702, p=.032, M S_{\mathrm{c}}=0.061$. Block order did not result in a main effect $(F<1)$, nor did it significantly interact with support, $F(1,14)=1.929, p=.187$, $M S_{\mathrm{e}}=0.061$.

Experiment 3 replicated the results of Experiments 1 and 2; subjects showed a consistent tendency to misremember the position of an object as farther down from true-same when the trial began with a display showing clear support for the object, but not when the initial display did not show clear support. Experiment 3 extended the results of the first two experiments by using a new stimulus display, by comparing support and no-support conditions within subjects, and by looking at a range of distractor positions centered around 


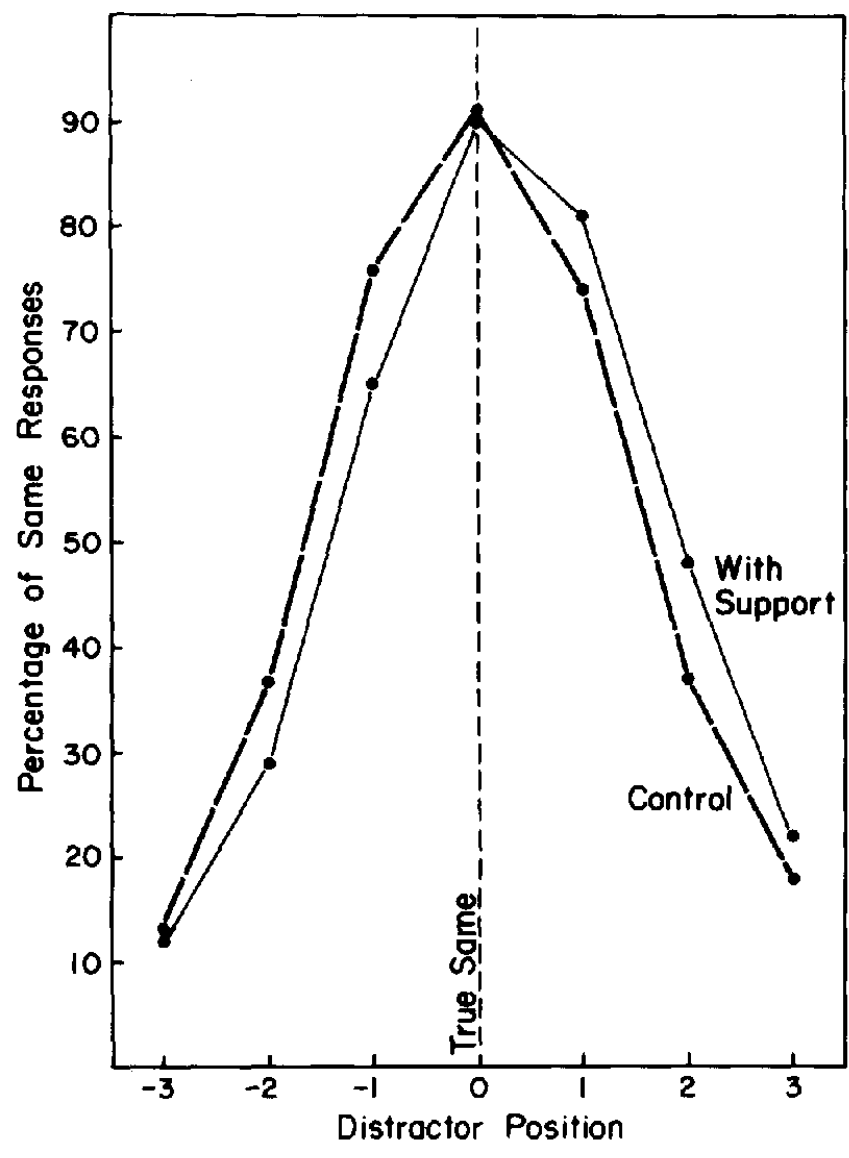

Figure 5. Percentage of same responses for the support-present (With Support) and no-support (Control) conditions of Experiment 3 are plotted for each of seven distractor positions. Negative distractor positions indicate lock positions above true-same (the 0 distractor position); positive distractor positions indicate lock positions below true-same.

true-same. This last feature of Experiment 3 allows us to see that the memory errors for the no-support condition are symmetric with respect to true-same, as the errors for the support-present condition are symmetric with respect to a position downward from true-same (see Figure 5).

The results of Experiment 3 might seem to differ from some of the previously reported results from representational momentum experiments using a range of distractor positions: We did not find what we refer to as a "recorded shift," that is, a case in which the number of same responses was greater for a true-different position than for true-same, as was found in a number of representational momentum experiments (e.g., Finke, Freyd, \& Shyi, 1986, Freyd \& Finke, 1985; Kelly \& Freyd, 1987). Instead, in Experiment 3 we found only a positive estimated shift for the support condition, based on differences between the number of same responses for different distractor positions (see also Cooper, Gibson, Mowafy, \& Tataryn, 1987). In previous studies (e.g., Freyd \& Johnson, 1987) in which recorded shifts were found for some conditions, and only estimated shifts for others, the distinction was not considered qualitative but quantitative. This assumption is supported by (a) the lawful relationship between estimated shifts for conditions varying in some parameter (e.g., retention interval in Experiment 2 in Freyd \& Johnson, 1987), independent of whether the individual conditions show a recorded shift and (b) the smooth, unimodal, and symmetric functions relating the number of same responses to distractor positions, as found for both conditions of Experiment 3. We believe that given a significant estimated shift, the finding of a recorded shift depends on using a range of distractor positions with sufficiently fine resolution. In either case we assume that the theoretical distribution of same responses is shifted such that it is symmetrically centered around some position different from true-same.

\section{Experiment 4}

In Experiments 1-3 we investigated the tendency to misremember objects as farther down than previously shown due to a representation of gravity. In Experiment 4 we investigated a different, more complex stable situation and predicted memory shift following the unbalancing of forces. We used displays involving a box resting on a coiled spring and, depending on the inducing display sequence, predicted that subjects would misremember the spring as more or less compressed than it actually was. With this display we hoped to learn whether the initial results generalized beyond the representation of simple gravitational force.

Figure 6 illustrates the two display sequences we used in Experiment 4 . In the box-added condition, a coiled spring was shown alone, followed by a second display of the spring with a box resting on it. In the box-removed condition, the coiled spring was shown initially with the box in place and then shown without the box. The spring compression in the first two displays was constant and the same for both conditions. We used seven distractor displays, with spring compression varied parametrically around true-same. As in the other experiments, we assumed that the second display would be interpreted as depicting the scene at the instant the equilibrium of the forces was disrupted. We thus predicted that subjects would misremember the spring as more compressed in the box-added condition and the spring as less compressed in the box-removed condition.

Experiment 4 allowed us to avoid two shortcomings of the previous experiments. First, we predicted an upward memory shift for one of the conditions. Such a finding would serve as further evidence against an alternative hypothesis for Experiments 1-3 that postulates some sort of general tendency to misremember objects as further down. Second, the to-beremembered displays in Experiment 4, unlike the first three experiments, depicted potentially stable scenes when viewed alone. Thus any memory shift found would have to be dependent on the display sequence.

\section{Method}

Subjects. Twenty-four members of the Cornell University community were paid for their participation in this experiment. The first 12 subjects were tested in the box-removed condition; the second 12 subjects were tested in the box-added condition. None had participated in Experiment 1, 2, or 3.

Apparatus. The apparatus was the same as in the first three experiments. 


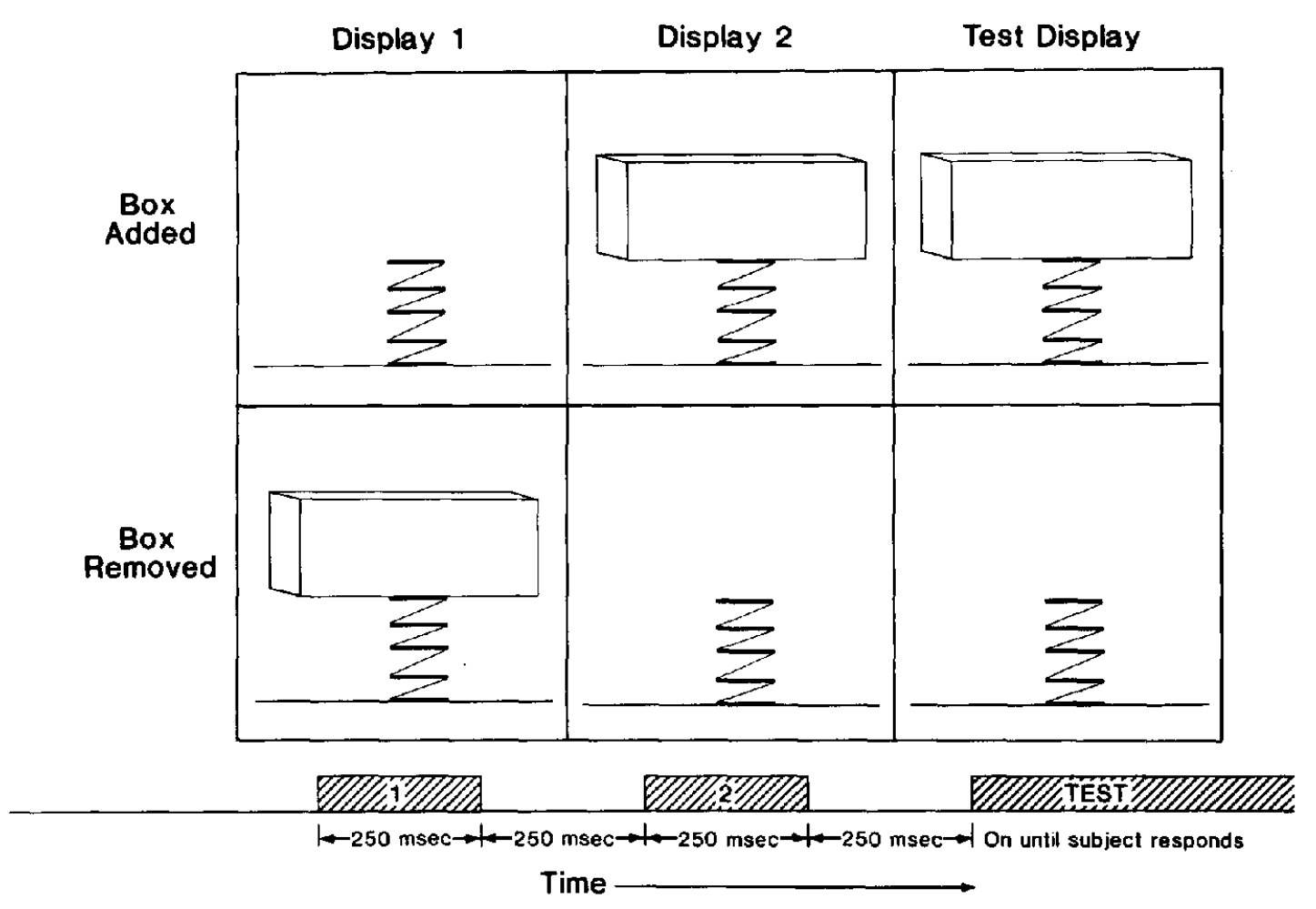

Figure 6. Schematic depiction of the display sequences used in each of the two conditions of Experiment 4.

Stimuli and format of trials. The timing sequence for each trial was the same in the present experiment as in Experiments 1-3. The display sequences used in Experiment 4 are depicted schematically in Figure 6. In the box-removed trials, subjects were shown first a drawing of a spring with a box resting on it and then that same spring without the box in the second display. In the box-added trials, the order of presentation of the first two displays was exactly the reverse of box-removed trials; subjects were shown first a spring alone and then a spring with a box resting on it. The first two displays were identical for the two conditions except that the order of presentations was reversed.

The first two displays in each trial showed a horizontally centered spring with a fixed compression; that is, the spring did not change with the addition or removal of the box. The bottom of this standard spring was $0.61 \mathrm{~cm}$ from the bottom of the screen and coincided with a slightly lower intensity horizontal straight line indicating a resting surface for the spring. The standard spring was $1.69 \mathrm{~cm}$ in length, formed of five equally spaced horizontal lines and four connecting diagonal lines drawn with a slightly lower intensity. An inspection of Figure 6 shows that these horizontal and diagonal lines appear to be a group of connected coils forming a spring viewed from the front.

The box, which was shown resting on the spring, was an approximate perspective drawing of a three-dimensional block showing front, left side, and top faces. The intensity of the lines forming the box was lower than the intensity of the horizontal lines forming the coils in the spring. The front face was $3.76 \mathrm{~cm}$ wide by $1.74 \mathrm{~cm}$ tall. The line indicating the back edge of the left side was $0.56 \mathrm{~cm}$ on the surface of the screen from the left edge of the front face. The line indicating the back edge of the top was $0.09 \mathrm{~cm}$ on the surface of the screen from the top edge of the front face.

The seven test displays were created by parametrically varying the compression of the spring around the standard spring. In the boxadded condition, the test springs were shown with the box present; in the box-removed condition, the test springs were shown alone. The seven test displays were otherwise identical for the two conditions. The bottom of the spring was anchored; the top of the spring was raised or lowered in steps of $0.07 \mathrm{~cm}$ to create the seven distractor positions. Compression was then manipulated by equally shrinking or expanding the distance between the five horizontal lines forming the coils and by adjusting diagonal lines appropriately.

Procedure. Subjects received thorough written and oral instructions very similar to the instructions used in the first three experiments, except that instead of emphasizing memory for position, they emphasized memory for the exact appearance of the spring or springbox combination. Similarly, individual trials followed the timing and ordering used in the first three experiments. In this experiment subjects received one critical block of 84 trials, preceded by 14 practice trials. The 14 practice trials were formed of 2 replications of each of the seven test positions. The 84 critical trials were formed of 12 replications of each of the seven test positions. For the first 12 subjects, the critical block and practice trials contained the display sequence for the box-removed condition; the remaining 12 subjects were presented with box-added trials. Condition was thus a between-subjects variable.

While subjects were in the laboratory, they were asked to complete additional blocks of trials, for related experiments with a similar paradigm, as in Experiments 1 and 2. The order of blocks was counterbalanced across subjects for each condition; preliminary analyses indicated that ordinal position of the block within the experimental session did not interact with any factors of interest.

\section{Results and Discussion}

The percentages of same responses were calculated for each of the seven test positions for each subject. The top half of 
Table 2 shows mean percentage of same responses presented separately for the 12 subjects in the box-added condition and the 12 subjects in the box-removed condition.

Because we were unsure of the correct relationship between the ordinal characterization of distractor positions $(-3$ to +3$)$ and the physical characterization of spring compression (for which there is a choice of measuring compression by using overall length of spring, change in length, change in angle at each coil, etc.), we did not calculate shift estimates by first subjecting the data to quadratic regressions (in which a linear scale is assumed for the predicting variable) as in Experiment 3. Instead we used the number of same responses for the -1 and +1 test positions in analyses comparable to those performed for Experiments 1 and 2. Figure 7, Section A displays the mean percentage of same responses for the -1 (less compressed) and +1 (more compressed) test positions for each of the two conditions.

Our first analysis was a two-way ANOva in which condition (box added or box removed) was a between-subjects variable and direction of compression (more or less) was a withinsubjects variable. The main effect for condition was not significant: $F(1,22)=.844, p=.368, M S_{\mathrm{e}}=7.135$. Similarly, the main effect for direction of compression was not significant: $F(1,22)=.005, p=.946, M S_{\mathrm{e}}=4.407$. The crossover interaction between condition and direction of compression was highly significant in the predicted direction: $F(1,22)=$ $16.455, p=.001, M S_{\mathrm{e}}=4.407$.
Table 2

Experiment 4 and Replication: Percentage of Same

Responses Between Subjects and Within Subjects

\begin{tabular}{|c|c|c|c|c|c|c|c|}
\hline \multirow[b]{3}{*}{ Condition } & \multicolumn{7}{|c|}{ Compression of test display } \\
\hline & \multicolumn{3}{|c|}{ More } & \multirow[b]{2}{*}{0} & \multicolumn{3}{|c|}{ Less } \\
\hline & +3 & +2 & +1 & & -1 & -2 & -3 \\
\hline \multicolumn{8}{|c|}{ Experiment 4 (between subjects) } \\
\hline $\begin{array}{l}\text { Box removed } \\
\text { Box added }\end{array}$ & $\begin{array}{r}9 \\
12\end{array}$ & $\begin{array}{l}19 \\
40\end{array}$ & $\begin{array}{l}51 \\
78\end{array}$ & $\begin{array}{l}81 \\
81\end{array}$ & $\begin{array}{l}72 \\
58\end{array}$ & $\begin{array}{l}31 \\
28\end{array}$ & $\begin{array}{l}17 \\
15\end{array}$ \\
\hline \multicolumn{8}{|c|}{ Replication (within subjects) } \\
\hline $\begin{array}{l}\text { Box removed } \\
\text { Box added }\end{array}$ & $\begin{array}{r}9 \\
17\end{array}$ & $\begin{array}{l}36 \\
43\end{array}$ & $\begin{array}{l}65 \\
80\end{array}$ & $\begin{array}{l}83 \\
86\end{array}$ & $\begin{array}{l}77 \\
71\end{array}$ & $\begin{array}{l}29 \\
38\end{array}$ & $\begin{array}{l}15 \\
13\end{array}$ \\
\hline
\end{tabular}

Note. Negative distractor positions indicate springs that are less compressed than true-same (the 0 distractor position); positive distractor positions indicate springs that are more compressed than truesame. The higher the absolute value of the distractor position, the larger the physical dissimilarity from true-same.

In our second set of analyses, we looked separately at the box-removed and box-added conditions. A $t$ test on the boxremoved results revealed that the percentage of same responses for the less compressed distractor position (the -1 test item) was significantly different from the percentage of same responses for the more compressed distractor position (the +1 test item): $t(11)=2.83, p=.016$. Similarly, a test on

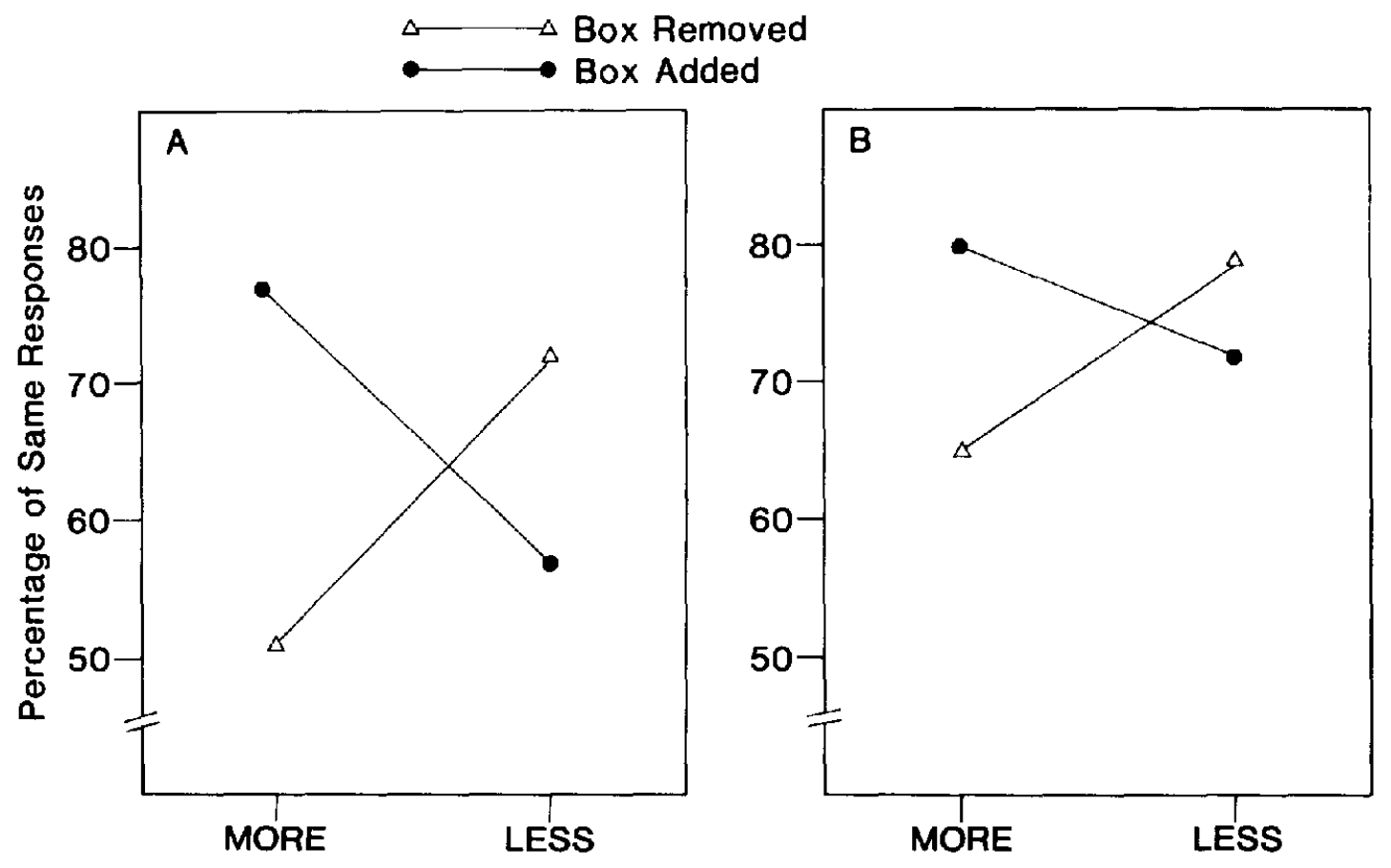

Distractor Compression

Figure 7. Section A: Percentage of same responses for the box-added and box-removed conditions of Experiment 4 are plotted for the closest more distorted and less distorted test positions (Position +1 and -1 , respectively, in Table 2). Section B: Percentage of same responses for the box-added and boxremoved conditions of the within-subjects replication of Experiment 4. 
the box-added results showed that the percentage of same responses for the less compressed distractor position was significantly different from the percentage of same responses for the more compressed distractor position: $t(11)=2.91, p$ $=.014$. These significant differences were in the predicted direction and consistent with the significant crossover interaction discovered in the two-way analysis reported.

In a follow-up for Experiment 4, we attempted to replicate the results by using an entirely within-subjects design. Twelve subjects were recruited from the University of Oregon community. The equipment, stimuli, and procedure were unchanged from Experiment 4 except that subjects participated in both the box-added and box-removed conditions. (Conditions were blocked, and block order was counterbalanced across subjects.) The bottom half of Table 2 and Figure 7, Section B show that the within-subjects version of Experiment 4 replicated the between-subjects version very closely. A twoway ANOVA on the results of the replication revealed a significant interaction between condition (box added or box removed) and direction of compression (more or less): $F(1,11)$ $=6.341, p=.029, M S_{\mathrm{e}}=3.157$.

Experiment 4 extended the results of the first three experiments by showing that under appropriate conditions memory for the observed compression of a spring can be distorted in the direction one would predict given the sudden disruption of equilibrium. This extension suggests that the representation of forces includes cases that are more complex than the simple cases involving only gravity in the first experiments. In addition, the result for the box-removed condition (in which the memory asymmetry favors the less compressed spring) demonstrated that memory shifts can go in the upward direction as well as the downward direction (in becoming less compressed in these displays, the top of the spring moves upward).

\section{General Discussion}

In the experiments reported here, we found a consistent asymmetry in memory for the position of objects following indications that stabilizing forces were removed. We did not find the memory asymmetry when subjects initially viewed an unstable scene, followed by a second exposure of that unstable scene, suggesting that the effect depends on an initial representation of forces in equilibrium. We found that the effect generalized to cases in which the disruption of equilibrium changed the compression of a spring.

These results support the hypothesis that people represent some of the underlying physical forces in static scenes, although strictly speaking our effect measures represented instability, not stability, as it depends on the disruption of equilibrium. In future studies we hope to get a better measure of represented forces when those forces are in equilibrium. We also plan to look closely at the time course of the memory shift for the sort of displays used in the current experiments; if the memory shift grows lawfully during the retention interval (as was found for representational momentum, Freyd \& Johnson, 1987), we will have better evidence of dynamic representations for static scenes (see Freyd, 1987). In the meantime we take these results as supportive of the hypothesis that motivated these studies: The perceptual system includes forces in equilibrium in the representation of static scenes.
We conclude this article by discussing four questions related to our findings.

\section{How Do the Current Experiments Relate to Similar Experiments With Frozen-Action Photographs or Sequences of Static Displays?}

The current experiments are clearly similar in format and results to the experiment with frozen-action pictures reported by Freyd (1983) and to many of the representational momentum experiments (e.g., Freyd \& Finke, 1984). As in those experiments, subjects were asked to hold a static display in memory for a short time and then to make a same-different discrimination when presented with a test display. Also similar to the previous studies, our new results indicate a memory asymmetry in the direction predicted by a hypothesis implying that dynamic information is represented. However, there are some critical differences between the newer and older studies.

First, we consider the current experiments in contrast to Freyd's (1983) experiment with frozen-action photographs. The primary point of contrast is the manner in which mental transformations of the critical object (the object in the picture that does or does not change in some way) are induced. In the earlier study photographs were selected on the basis of being compelling indicators of unidirectional motion. The motion was richly implied by a variety of factors in the picture, such as deformation of shape and blur. In the current study only computer-generated line drawings were used, without rich indications of motion, and we relied on the sequence of displays to induce mental transformations. In the first three experiments, we used a drawing of an object suspended in midair as our to-be-remembered display; it is possible that such a display shown alone (e.g., if we had started our trials with the second display) would in itself induce a representation of motion. Arnheim (1974), Gombrich (1964), and Gottlieb (1958) have each proposed that a compelling sense of motion comes from viewing a painting that uses imbalance or instability in its placement of objects. We know, however, that the motion was not so compelling as to lead to a memory asymmetry when the object was shown unsupported in the first display as well as in the second. Furthermore, in Experiment 4 , in which a spring was shown, the single display of the spring was shown in a stable and supported context, and it alone should not have led to any memory asymmetries (this is indicated too by the fact that there was no main effect for direction of compression in Experiment 4). Thus, the memory asymmetry in the current experiments differs from the effect in the frozen-action experiments in depending on a sequence of static scenes.

The reliance on a sequence of static scenes to induce a memory asymmetry makes the current effect seem similar to representational momentum. In the representational momentum experiments, the direction of motion is given in the sequence of static positions sampled from a transformational path. Perhaps even more so than in the frozen-action experiment, the direction of motion is clearly implied. In contrast, change was implied in the present study by changing implied forces: by removing a source of support (Experiments 1 and 3) or by adding or removing a weight resting on a spring (Experiment 4), while specifically displaying the critical object 
as unchanged in the inducing sequence. In this way the present studies are different from the sequence of static displays used in representational momentum studies in which the critical object is shown in different positions.

Despite these differences between the current experiments and the earlier studies, we believe that the effects stem from a common underlying representational system that emphasizes dynamic information. In all these studies a memory asymmetry arises following a very brief retention interval (a fraction of a second) that is predicted by the implied motion (or changes in balance of forces) of the display. As discussed by Freyd (1987), we hypothesize that the representations serving perception have a fully integrated temporal dimension that cannot be extracted even when it would be expedient to do so (as when attempting to remember a static position) and that the observed memory asymmetries reflect this property of perceptual representations. The current studies extend the past research by suggesting that the representation of dynamics might include perception of static scenes.

\section{What Is the Nature of the Represented Force Dimension?}

At present we do not know much about the mental representation of physical forces. Force can be conceptually decomposed in various ways, and it is possible that our representation of force reduces to the representation of a number of separate dimensions. Our theoretical perspective leads us to believe that the representation of force emphasizes the underlying temporal aspect of force. The classic decomposition of force is to express force as the product of mass and acceleration. A temporal dimension is clearly embedded in acceleration.

An alternative conceptualization of force that may map more naturally onto our notion of dynamic mental representations (Freyd, 1987) is to express force as the rate of "momentum flow." (These two analyses reduce to the same thing.) DiSessa (1980) argued that it is intuitively difficult to understand the role of force as mass times acceleration in many static scenes. For instance, when using a classic force analysis to understand why an apple resting on a hand is static, one must understand that the hand is providing a force equal to and opposite that of gravity. DiSessa (1980) claimed that the action-reaction relationship is difficult for people to understand, making the classic force analysis on the resting apple intuitively complex. In contrast, a momentum flow analysis sees gravity as flowing into the apple, flowing from the apple to the hand, from the hand to the body, and then to all the places in the earth where the momentum flow originated in the first place. We see the momentum flow analysis as natural for dynamic mental representations, at least at an intuitive level, because it stresses the continuous flow of something even when no motion is present.

However, we are probably not at a point in our investigation of dynamic mental representation to specify the nature of the force dimensions in the underlying representation. We first need to determine whether the perceptual system is sensitive to the magnitude of forces as opposed to sensitive simply to the direction (such as knowing that gravity pulls down and springs push and pull). One reason to hypothesize that there is sensitivity to magnitude is that the amount of representational momentum is determined by the implied acceleration of a display and thus the implied final velocity, not the average velocity (Finke et al., 1986); sensitivity to acceleration is probably necessary for sensitivity to the magnitude of a force. In future research we hope to determine how sensitive the perceptual system is to the magnitude of forces in equilibrium.

Perhaps we might also be able to determine whether the present findings generalize to physical situations beyond gravity, such as those where pressure (or even electromagnetic force) dominates. However, we suspect that gravity is a better candidate for mental "internalization" than other forces. Shepard $(1981,1984)$ has argued that the mind has internalized characteristics of the world that have been most pervasive and enduring throughout evolution. Although Shepard's (1981, 1984) list has emphasized kinematic, as opposed to dynamic, transformations, the dynamic aspects of gravity are indeed pervasive and enduring characteristics of the world.

\section{Does the Representation of Forces Help Solve Problems Confronting the Perceptual System?}

A major issue for students of object recognition is how people can correctly identify a particular instantiation of an object given that they have probably never previously viewed the object from exactly the perspective held at a given moment. One solution to this problem has been to suggest that the mind represents possible transformations an object could undergo (typically the rigid transformations of rotation and translation that map onto different viewing perspectives) along with a particular instantiation. This solution has been suggested in different forms, for instance, by Cassirer (1944), Helmholtz (1894/1971), and Shepard (1981). Our current results suggest that not all possible transformations are necessarily equal in the representation of a static object. In particular, some of those transformations that correspond to plausible unbalancings of forces might be more available than others. We plan to explore the possibility that the transformations represented for an object may include those that follow from likely additional forces (such as forces created by the observer in order to move the object) as well as transformations created by the likely removal of forces.

Having some sort of access to likely transformations by representing physical forces may help solve a slightly different problem in object recognition: the problem of correctly identifying a particular instantiation, or "token," as a member of a larger class, or type, of object. If part of what one stores in memory about an object type is aspects of its likely behavior when embedded in events, then representing physical forces operating on objects in a particular perceptual situation may help in the process of identification of a particular object token.

Whether the representation of physical forces aids object recognition or not, it surely would be useful for event perception, in which change over time must be correctly registered by the perceptual system. An accurate representation of physical forces will constrain the perception and, most important, the anticipation of events; the representation of forces in event perception allows the system to move from visually presented kinematics to underiying dynamics (Todd \& Warren, 1982). 
The representation of forces should similarly aid in planning motor behavior when the observer wishes to interact with moving or stable objects. Consider the problem posed to the perceptual-motor system whenever it is in the process of moving an object. Not only must the system determine the correct location in space and time for grasping, but it must also determine the correct force with which to grasp and lift or push and so forth (see Iberall, 1986). This depends critically on the dynamic qualities of the object, including the forces operating on the object. Of course, to go correctly from visual input to a representation of forces, the underlying representational system has to "know" something about physical forces and how they interact with objects for a particular environment, such as the environment encountered on the surface of the planet Earth. Such knowledge may be a function of the inherited or experientially modified representational structure serving perception.

\section{What is the Locus of the Representation of Forces: Perceptual or Conceptual?}

There is a potential discrepancy between our hypothesis that forces are represented in static scenes and the common introspection that the information available to consciousness while a person is viewing a static scene does not suggest such a representation of forces. Unless schooled in physics, most people are surprised to learn that forces operate on static objects. Our response to this discrepancy is twofold. First, we suggest that there is a phenomenal sensation that suggests that forces are represented even in stable scenes, and that is the phenomenal sense of concreteness. When objects look firmly planted, or even heavy, people may be relying on a representation of forces to arrive at that conscious sensation. If we are correct, then the conscious perception of concreteness, which can occur with entirely visual input, may stem from the underlying representation of forces, even if people are not conscious of such representations.

Our second response to the apparent discrepancy between our hypothesis and conscious experience is to propose that our experimental findings, taken together with related findings, implicate perceptual knowledge of physical laws, not conceptual knowledge. This line of argument is complicated by the fact that although our motivating hypothesis (that forces are represented when static scenes are viewed) may be apparently discrepant with conscious experience, our experimental findings are apparently consistent with common conceptual knowledge: Most people can explicitly state that if a table supporting a plant is suddenly removed, the plant will most likely fall or that if a box resting on a spring is suddenly removed the spring will most likely become less compressed. Despite this apparent consistency between our findings and conceptual knowledge, we believe that our effect results from the nature of the perceptual system, not from explicit or conscious knowledge available to more central processing (Fodor, 1983). One reason for this assumption of perceptual modularity is that it follows from our motivating hypothesis: Although we induce our effect by implying sudden disequilibrium, our motivating hypothesis, that forces are represented while a person is viewing static scenes, is discrepant with common conceptual knowledge. The relationship between our findings and our hypothesis would be called into question if it turned out that the memory effects stemmed from conceptual knowledge of instability.

Our assumption of perceptual modularity is supported by the nature of the task we are using and by the findings relevant to modularity for representational momentum (see Kelly \& Freyd, 1987). Although we measure memory asymmetries (Fodor, 1983, considered memory to be central processing), it is memory for a display seen only $250 \mathrm{~ms}$ previous to the test display; we assume that this memory is part of the perceptual system and exists by virtue of perceptual representations. Our task asks that subjects simply remember what they saw, and any effect we measure is in spite of subjects' efforts to avoid transformations in memory. This suggests that the effect is mandatory, one of the properties that Fodor suggested is characteristic of informational encapsulated modular systems. A second property that Fodor considered diagnostic is very rapid processing. In the experiments reported here, the memory transformation presumably occurred in the 250-ms retention interval between the to-be-remembered display and the test display. From the related phenomenon of representational momentum, we have reason to assume that the processing is even more rapid than $250 \mathrm{~ms}$; looking at retention intervals ranging from $10 \mathrm{~ms}$ to $100 \mathrm{~ms}$ in $10-\mathrm{ms}$ steps, Freyd and Johnson (1987) found that the degree of memory asymmetry for the remembered position of a static object with an implied rotational motion increased linearly over the first $100 \mathrm{~ms}$ of the retention interval. The rapid and mandatory nature of representational momentum led Kelly and Freyd (1987) and Freyd (1987) to argue for the cognitive impenetrability of the effect. To the extent that our finding reflects the same sorts of representational properties and constraints as does representational momentum, we assume that it is cognitively impenetrable and that it stems from the perceptual system.

Although some might accept that the force of gravity and its simple opposing forces (Experiments 1-3) could be represented within the perceptual system, many might argue that the representation of forces active in springs (Experiment 4) implicates real-world learning and thus suggests that the basis of the effect is more central than perceptual. We suggest two responses to this argument: First, perceptual knowledge of springlike behavior may be innately given and not dependent on learning; second, evidence of perceptual learning is not necessarily evidence against modularity. For both of these responses, we question the assumption that the effect in Experiment 4 stems from knowledge of springs per se. It might instead reflect perceptual knowledge of compressible and elastic substances, of which springs are an example. DiSessa (1983) suggested that springiness is a phenomenological primitive. E. J. Gibson, Owsley, Walker, and MegawNyce (1979) found that 3-month-old infants extract object rigidity or nonrigidity from motion, suggesting that people distinguish compressible from noncompressible substances at a very early age. Learning about substances could certainly occur within 3 months, but even so that would not rule out the possibility that the knowledge was encoded within a perceptual module. That is, a modular input system could 
learn about its perceptual environment without compromising modularity as long as that learning modified the module itself. Evidence against modularity would come not from evidence of learning but from a demonstration of cognitive penetrability where central knowledge interrupts or interferes with ongoing perceptual processing.

Other researchers have argued that there may be a difference between conceptual and perceptual knowledge about physical regularities. For instance, Shanon (1976) demonstrated that knowledge of gravitational acceleration is much more accurate when probed perceptually (as in a person watching a natural or anomalous film of an object falling) than when probed conceptually. Kaiser, Proffitt, and Anderson (1985) similarly demonstrated that knowledge of linear momentum in the absence of external forces is apparently more accurate when gauged by asking subjects to respond to filmed motions than when gauged by asking subjects to respond to paperand-pencil questionnaires (as in McCloskey, Caramazza, \& Green, 1980). Not only has the possibility of a discrepancy between perceptual and conceptual knowledge been suggested (although not agreed on by all; e.g., McCloskey, Washburn, \& Felch, 1983), some researchers have proposed that people can hold conflicting conceptual theories (e.g., see Roncato \& Rumiati, 1986, on the possibility that people hold conflicting beliefs about stable and neutral equilibrium). It seems likely to us that there are multiple, and often discrepant, levels of knowledge about physics in the human mind; our present interest is in the laws of force and motion embedded in the perceptual system.

In summary, we do not find it paradoxical that the perceptual system may represent forces in static scenes even though most people need education in mechanics to have explicit knowledge of forces in statics. Instead we believe that this apparent discrepancy reflects a distinction between knowledge embedded in the perceptual system and knowledge available to more central processing, along the lines of Fodor's (1983) modularity thesis. Indeed, our view suggests that when people are viewing a static scene, lurking behind the surface of consciousness is an inherently dynamic tension resulting from the representations of forces in equilibrium. We see this dynamic tension as contributing to the conscious experience of concreteness in perception and to the memory asymmetries we measure when the equilibrium is disrupted.

\section{References}

Arnheim, R. (1974). Ant and visual perception (the new version). Berkeley: University of California Press.

Cassirer, E. (1944). The concept of group and the theory of perception. Psychologica, 5, 1-35.

Cooper, L., Gibson, B. S., Mowafy, L., \& Tataryn, D. J. (1987, November). Mental extrapolation of perceptually-driven spatial transformations. Paper presented at the 28th Annual Meeting of the Psychonomic Society, Seattle, WA.

DiSessa, A. A. (1980). Momentum flow as an alternative perspective in elementary mechanics. American Journal of Physics, 48, 365369.

DiSessa, A. A. (1983). Phenomenology and evolution of intuition. In E. Gentner \& A. L. Stevens (Eds.), Mental models (pp. 15-33). Hillsdale, NJ: Erlbaum.
Finke, R. A., Freyd, J. J., \& Shyi, G. C.-W. (1986). Implied velocity and acceleration induce transformations of visual memory. Journal of Experimental Psychology: General, 115, 175-188.

Fodor, J. A. (1983). Modularity of mind. Cambridge, MA: MIT Press/ Bradford Books.

Freyd, J. J. (1983). The mental representation of movement when static stimuli are viewed. Perception \& Psychophysics, 33, 575-581.

Freyd, J. J. (1987). Dynamic mental representations. Psychological Review, 94, 427-438.

Freyd, J. J., \& Finke, R. A. (1984). Representational momentum. Journal of Experimental Psychology: Learning, Memory, and Cognition, 10, 126-132.

Freyd, J. J., \& Finke, R. A. (1985). A velocity effect for representational momentum. Bulletin of the Psychonomic Society, 23, 443446.

Freyd, J. J., \& Johnson, J. Q. (1987). Probing the time course of representational momentum. Joumal of Experimental Psychology: Learning, Memory, and Cognition, 13, 259-268.

Gibson, E. J., Owsley, C. J., Walker, A., \& Megaw-Nyce, J. (1979). Development of the perception of invariants: Substance and shape. Perception, 8, 609-619.

Gibson, J. J., (1970). On the relation between hallucination and perception. Leonardo, 3, 425-427.

Gibson, J. J. (1979). The ecological approach to visual perception. Boston: Houghton Mifflin.

Gombrich, E. H. (1964). Moment and movement in art. Journal of the Warburg and Courtland Institute, 27, 293-306.

Gottlieb, C. (1958). Movement in painting. Journal of Aesthetics and Art Criticism, 17, 22-33.

Helmholtz, H. von. (1971). The origin and correct interpretation of our sense impressions. In R. Kahl (Ed.), Selected writings of Hermann von Helmholtz (pp. 501-512). Middleton, CT: Wesleyan University Press. (Original work published 1894).

Iberall, T. (1986). The representations of objects for grasping. The proceedings of the 8th annual conference of the Cognitive Science Society, Amherst, MA.

Kaiser, M. K., Proffitt, D. R., Anderson, K. (1985). Judgments of natural and anomalous trajectories in the presence and absence of motion. Journal of Experimental Psychology: Learning, Memory, and Cognition, 11, 795-803.

Kelly, M. H., \& Freyd, J. J. (1987). Explorations of representational momentum. Cognitive Psychology, 19, 369-401.

McCloskey, M., Caramazza, A., \& Green, B. (1980). Curvilinear motion in the absence of external forces: Naive beliefs about the motion of objects. Science, 210, 1139-1141.

McCloskey, M., Washburn, A., \& Felch, L. (1983). Intuitive physics: The straight-down belief and its origin. Journal of Experimental Psychology: Learning, Memory, and Cognition, 9, 636-649.

Roncato, S., \& Rumiati, R. (1986). Naive statics: Current misconceptions on equilibrium. Journal of Experimental Psychology: Learning, Memory, and Cognition, 12, 361-377.

Shanon, B. (1976). Aristotelianism, Newtonianism and the physics of the layman. Perception, 5, 241-243.

Shepard, R. N. (1981). Psychophysical complementarity. In M. Kubovy \& J. R. Pomerantz (Eds.), Perceptual organization (pp. 279341). Hillsdale, NJ: Erlbaum.

Shepard, R. N. (1984). Ecological constraints on internal representation: Resonant kinematics of perceiving, imagining, thinking, and dreaming. Psychological Review, 91, 417-447.

Todd, J. T., \& Warren, W. H. (1982). Visual perception of relative mass in dynamic events. Perception, 11, 325-335.

Received December 14, 1987

Revision received May 9, 1988

Accepted May 11, 1988 